\title{
Parametric Analysis of the Improved Open Photoacoustic Helmholtz Cell
}

\author{
A. Geras - T. Starecki
}

Received: 13 April 2013 / Accepted: 29 July 2013 / Published online: 15 August 2013 (C) The Author(s) 2013. This article is published with open access at Springerlink.com

\begin{abstract}
Many photoacoustic cells use a Helmholtz structure for amplification of the photoacoustic signal, but very few of them are open cells. Open Helmholtz cells known from the literature exhibit very poor attenuation of the external acoustic noise, which severely limits their applications. Recent research showed that this problem can be overcome in a so-called improved open photoacoustic Helmholtz cell structure. The paper describes how mechanical dimensions of such a cell affect the frequency response of the cell and its penetration by the external acoustic noise. Properties of the cell were investigated by means of computer simulations based on the loss-improved transmission line model. The analysis showed that appropriate selection of the duct dimensions and buffer volumes should result in over $50 \mathrm{~dB}$ attenuation of the external acoustic noise, without noticeably affecting the $Q$-factor and resonance frequency of the cell.
\end{abstract}

Keywords External acoustic noise attenuation - Helmholtz resonator .

Open photoacoustic cell · Parametric analysis

\section{Introduction}

Open photoacoustic cells are often used in gas measurement equipment [1-5]. They are particularly suitable for continuous flow measurements, due to easy gas exchange and mechanical simplicity. Moreover, elimination of gas sampling may significantly reduce the time of measurements [5]. The main disadvantage of the open cells is that

\footnotetext{
A. Geras · T. Starecki $(\bowtie)$

Institute of Electronic Systems, Warsaw University of Technology,

Nowowiejska 15/19, 00-665 Warsaw, Poland

e-mail: t.starecki@ise.pw.edu.pl
} 


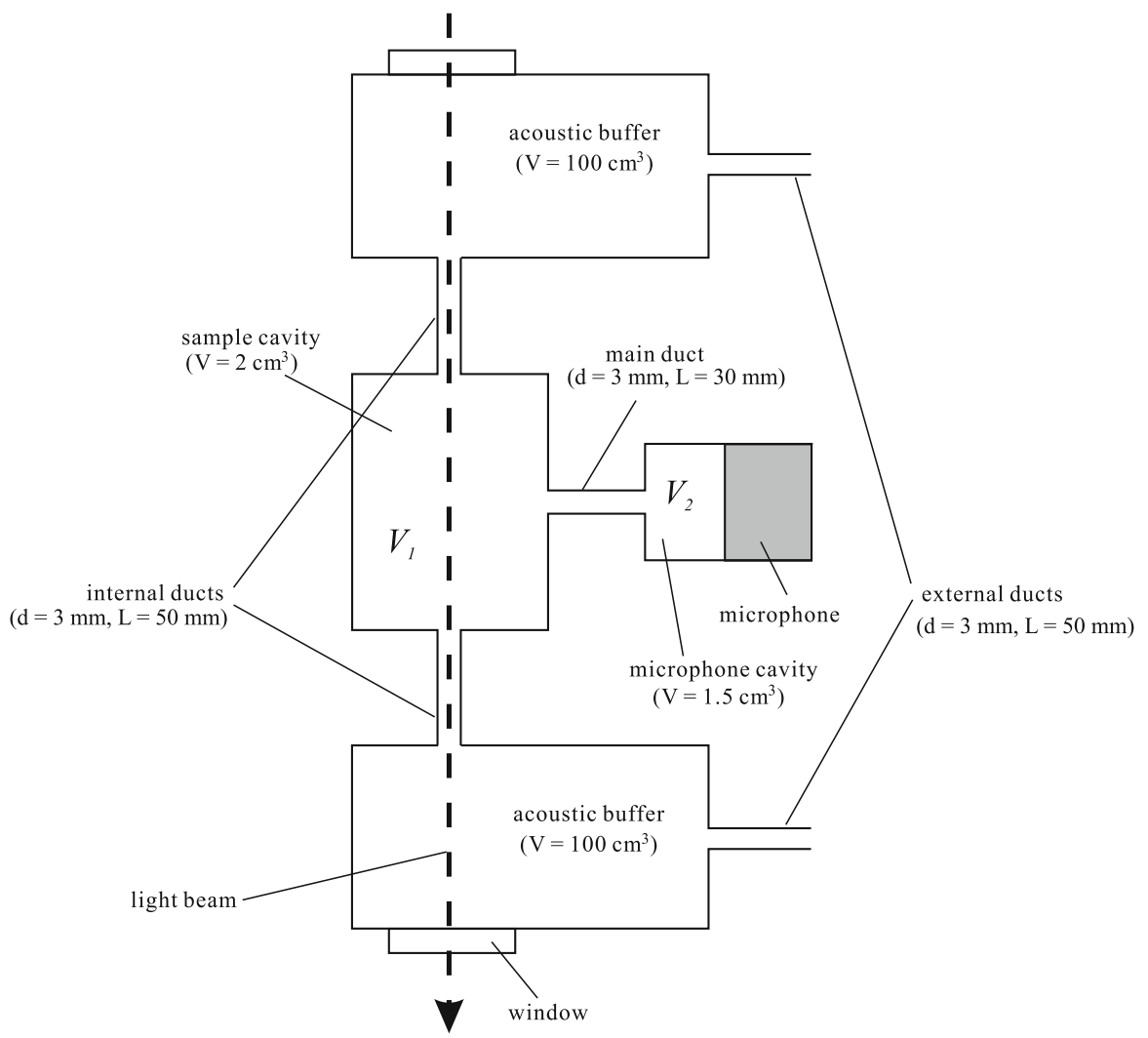

Fig. 1 Improved open photoacoustic Helmholtz cell design [9] with dimensions of the reference cell

penetration of the external acoustic noise is relatively strong, which may result in serious limitation of the sensitivity of the photoacoustic setup [6].

Quite a number of photoacoustic cells use the Helmholtz resonator structure, but very few of them are open cells. Solutions presented by Diószeghy et al. [7] and Starecki [8] have no or very limited applications due to amplification of the external acoustic noise at the frequency of resonance. However, in the case of an improved open Helmholtz cell (Fig. 1), which is a modification of an earlier design (Fig. 2) presented by Starecki [8], use of additional buffers reduces infiltration of the external acoustical noise by over $40 \mathrm{~dB}$, without affecting the frequency response at frequencies above $200 \mathrm{~Hz}$ (Fig. 3) [9]. Such properties mean that the cell can be considered for use in practical applications.

\section{Preliminary Considerations}

Properties of the improved open Helmholtz cell were investigated by means of computer simulations based on acousto-electric analogs [10,11]. In the implemented model of the cell (Fig. 4), acoustic cavities are represented by capacitors, and the ducts by 


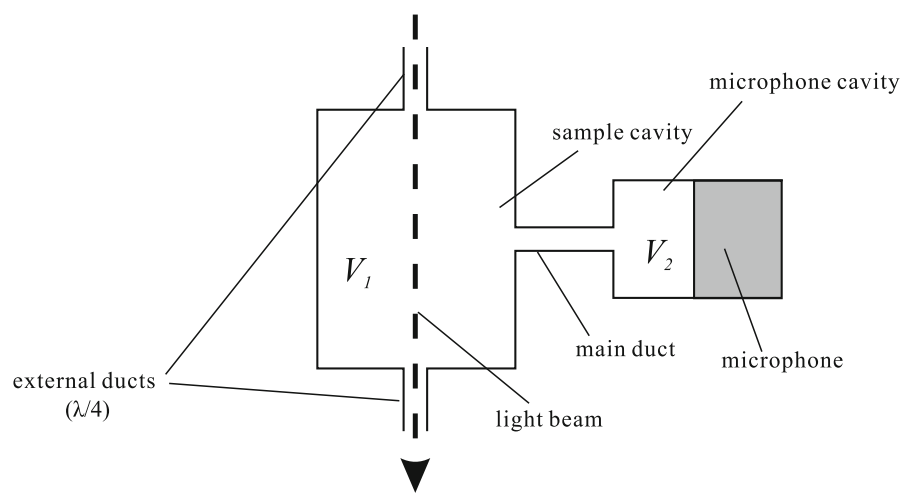

Fig. 2 Open photoacoustic Helmholtz cell [8]
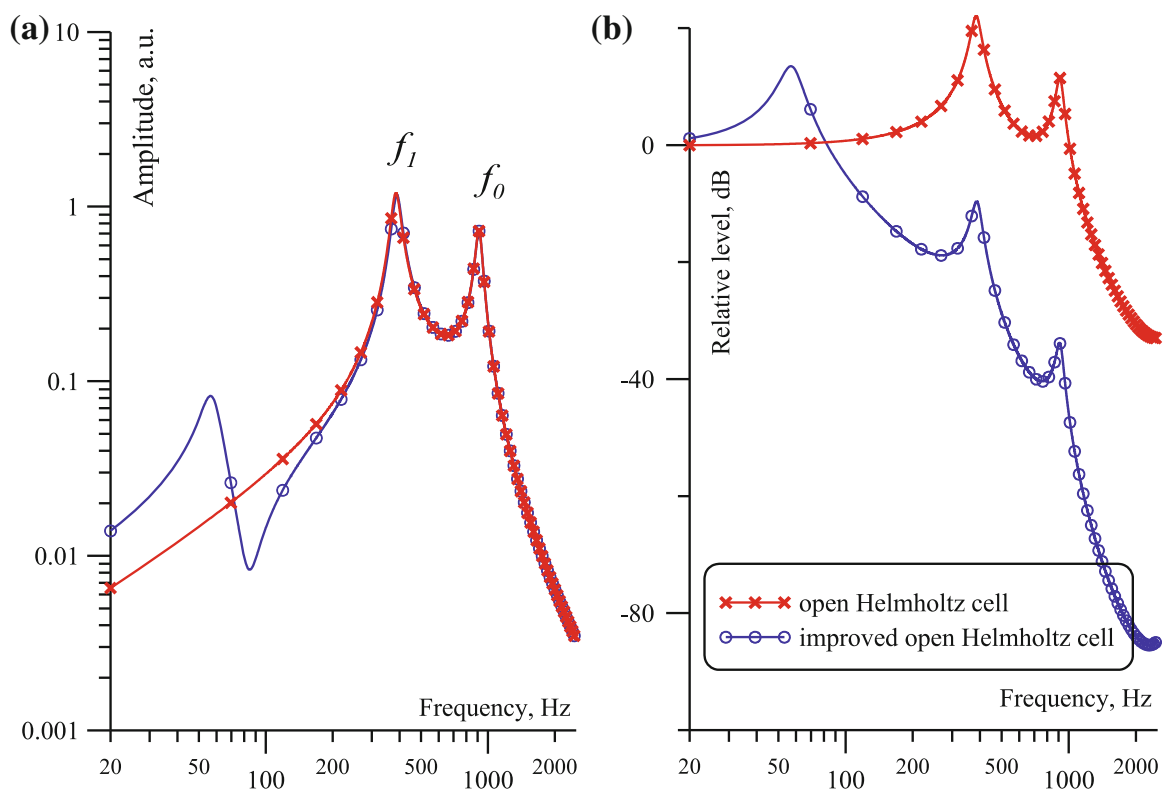

Fig. 3 (a) Frequency responses and (b) external noise infiltration of the open photoacoustic Helmholtz cell from Fig. 2 and improved open photoacoustic Helmholtz cell from Fig. 1 [9]

transmission lines converted into corresponding T-section impedances. The photoacoustic signal is introduced by the current source $I$ (definition of the current source should take into account that the amplitude of the induced photoacoustic signal is inversely proportional to the light modulation frequency), and the external acoustic noise by the voltage sources $U_{\mathrm{AL}}$ and $U_{\mathrm{AR}}$. Modeling of the exterior by means of two capacitances $C_{\mathrm{AL}}$ and $C_{\mathrm{AR}}$ (instead of single $C_{\mathrm{A}}$ ) and the external acoustic noise by two identical voltage sources $\left(U_{\mathrm{AL}}\right.$ and $\left.U_{\mathrm{AR}}\right)$ substantially simplifies calculations without affecting the final results. Pressure changes in the sample cavity are reflected by the $U_{\mathrm{S}}$ and in the microphone cavity by the $U_{\mathrm{M}}$ voltage signals. In such a case, the 


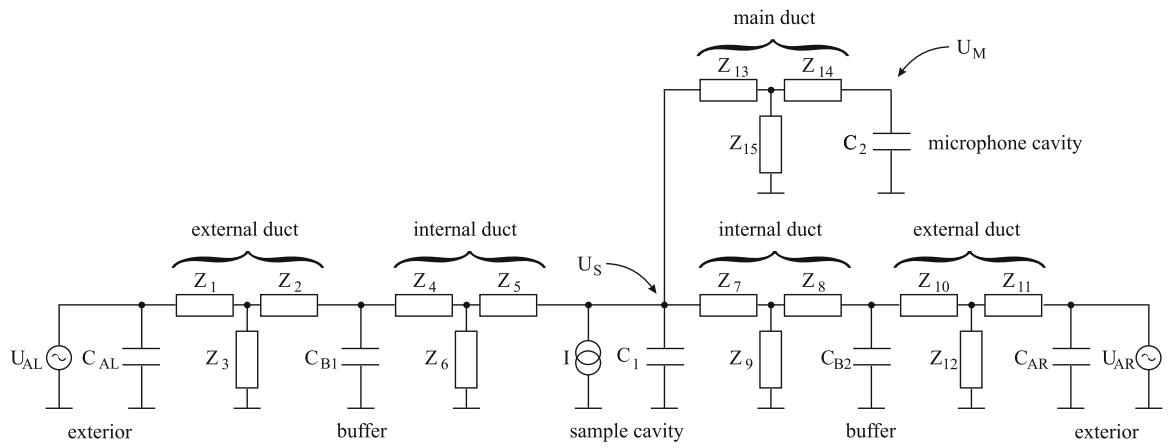

Fig. 4 Model used for determination of the properties of the improved open photoacoustic Helmholtz cell

frequency response of the cell is expressed by the amplitude of the $U_{\mathrm{M}}$ signal versus frequency, while the external acoustic noise penetration level is equal to the $U_{\mathrm{M}} / U_{\mathrm{AL}}$ ratio (where $U_{\mathrm{M}}$ is calculated as a superposition of the noise signals coming from the two mentioned voltage sources, $\left.U_{\mathrm{AL}}=U_{\mathrm{AR}}\right)$.

Although it is possible to describe acoustic ducts in a much simpler manner (as lossy inductances), use of transmission line counterparts results in better evaluation of thermal and viscous losses. In order to further improve the quality of the modeling, all the ducts were defined as in the loss-improved transmission line model [12,13], which had been previously employed in modeling of multicavity cells of similar dimensions. Results of these simulations were in very good agreement with the measurements $[8,12]$, so it can be assumed that the implemented model from Fig. 4 should give reliable information about the behavior of the investigated cell.

Preliminary simulations of the frequency response and external acoustic noise infiltration presented in Fig. 3 were obtained for a cell with the structure as in Fig. 1, in which the size of the sample and microphone cavities, as well as diameters and lengths of the main and internal ducts were identical as in the previously studied cell structure from Fig. 2 [8]. This allowed for easy comparison of the properties of the original cell and its improved version. A volume of $100 \mathrm{~cm}^{3}$ for the acoustic buffers is approximately two orders of magnitude greater than the sample cavity volume, so the acoustic impedance of the buffers can be considered as low. In such a case, quarter-wave ducts between the exterior and the buffers act as high-impedance components, substantially limiting external noise infiltration. With the modulation frequency adjusted to the cell resonance of approximately $900 \mathrm{~Hz}$, the calculated quarter-wave length would be $95 \mathrm{~mm}$. However, in the case of a small diameter, it may be difficult to produce ducts of such a length. In addition, previous investigations showed that although a quarterwave length is optimal, but making the duct shorter does not dramatically influence the $Q$-factor, the resonance frequency, or the noise properties of the cell [6]. For these reasons, the length of the external and internal ducts in the improved cell was set to $50 \mathrm{~mm}$, and their diameter to $3 \mathrm{~mm}$.

On the frequency response (Fig. 3a), two resonance peaks can be observed-at approximately $900 \mathrm{~Hz}$ and $300 \mathrm{~Hz}$. The first one, denoted with symbol $f_{0}$, results from the Helmholtz resonance between the sample and microphone cavities and will 

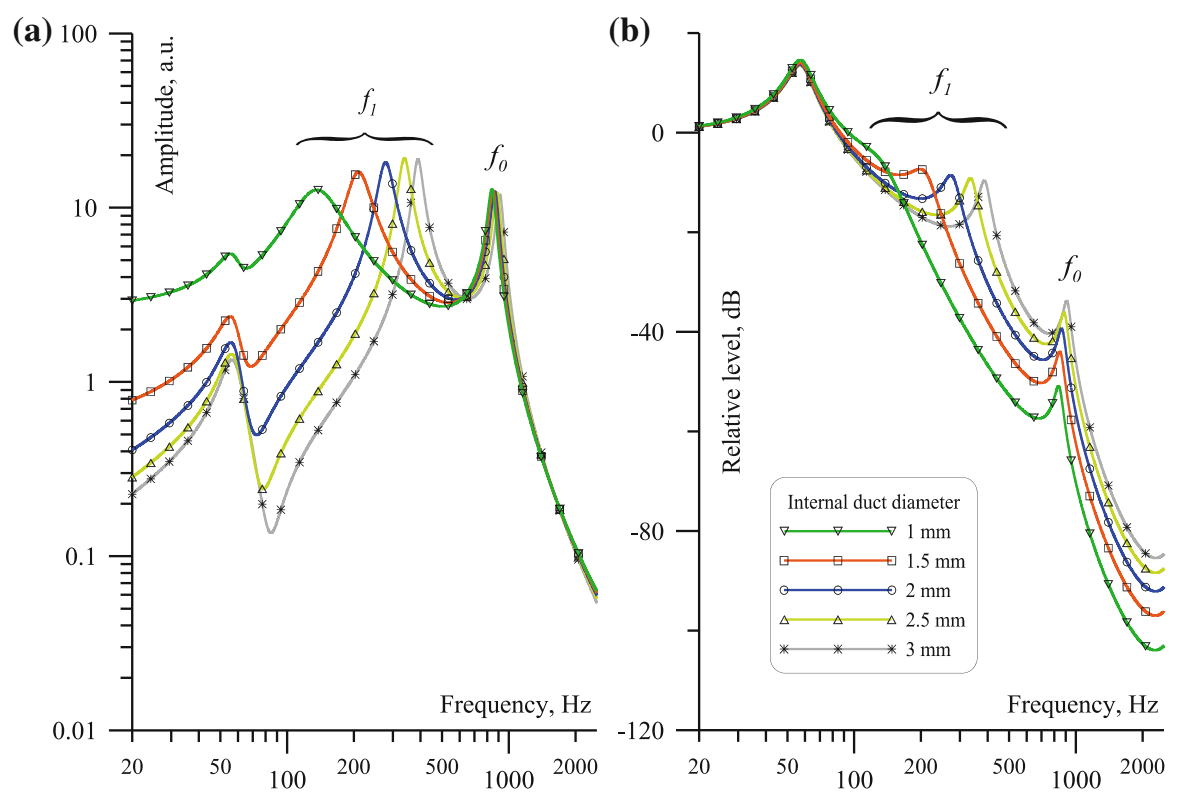

Fig. 5 Influence of the internal duct diameter on (a) the frequency response and (b) external acoustic noise infiltration of the cell

be called the main resonance. The second Helmholtz resonance (denoted with $f_{1}$ ) is a consequence of interaction between the cell and the exterior. It can be easily concluded from Fig. $3 \mathrm{~b}$ that noise infiltration at $f_{0}$ is much lower than at $f_{1}$. Therefore, $f_{0}$ rather than $f_{1}$ should be selected as the light modulation frequency (assuming that a cell resonance is used to amplify the photoacoustic signal).

\section{Modeling Results}

In order to determine the influence of mechanical parameters (diameter and length of the ducts, volume of the buffers) of the improved open Helmholtz cell on its operation, a number of computer simulations were performed. In every step only one of the mentioned dimensions was changed, while the remaining were the same as in the reference cell presented in Fig. 2. The results are discussed below.

As can be seen in Fig. 5a, internal duct diameters have a noticeable influence on the frequency response. For the given cell structure, a change of the diameter from $1 \mathrm{~mm}$ to $3 \mathrm{~mm}$ in an approximately $100 \mathrm{~Hz}$ change of the resonance frequency $f_{0}$ and an even greater change of $f_{1}$; however, the amplitudes of both resonances remain almost constant. External noise penetration can be significantly weakened by the reduction of the internal duct diameters (Fig. 5b). Unfortunately, in practical applications the internal duct diameter cannot be very small, because passing the light beam through the cell without irradiating the cell walls would be then very difficult, if possible. The smaller is the diameter, the more accurate must be collimation and positioning of the light beam, as well as the mechanical precision of the cell manufacturing. 

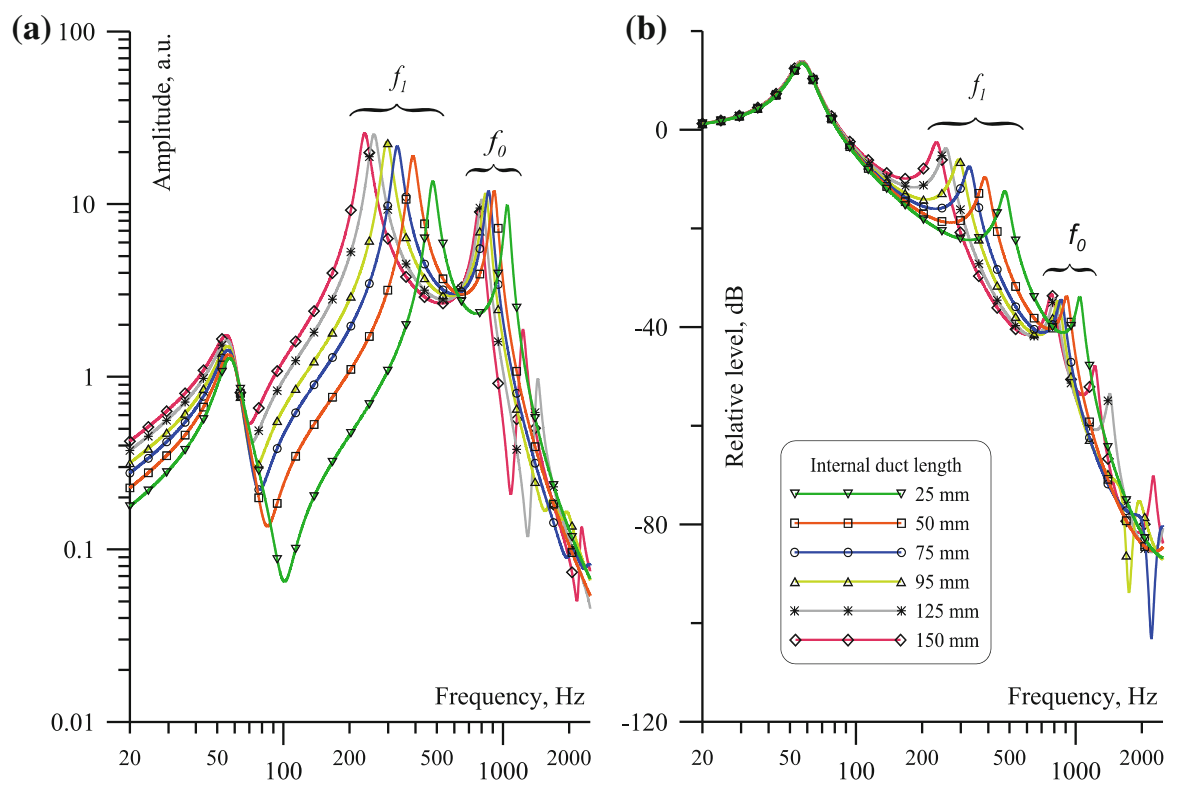

Fig. 6 Influence of the internal duct length on (a) the frequency response and (b) external acoustic noise infiltration of the cell

The length of the internal ducts contributes to the overall optical path. Similarly, as in the case of a very small internal duct diameter, an increase in the length of these ducts makes it more difficult to assure the light would not interact with the walls.

It is clearly visible in Fig. 6a that the frequency response of the cell is strongly related to the internal duct length. The resonance frequencies increase against the length of the internal ducts. At a closer look, it can be noticed that there is an optimal internal duct length at which the amplitude at the $f_{0}$ resonance reaches a maximum value. Changes of the internal ducts length influence noise infiltration in a similar manner (Fig. 6b), i.e., there is an optimal duct length at which attenuation of the external acoustic noise at $f_{0}$ has maximum value.

The external ducts are inlets and outlets for the gas being measured, as well as for the external acoustic noise. Thus, their diameters affect the speed of gas exchange and external noise penetration. Obviously, a greater cross-section of the ducts means that noise can enter the cell more easily. Therefore, the ducts should be of the smallest, yet still producible, diameters. Taking into consideration that in the cell from Fig. 1 the external ducts are not on the optical path, their diameters can be reduced to any required value (on contrary to the previously discussed internal ducts). In the simulations, the diameters were changed in the range from $1 \mathrm{~mm}$ to $3 \mathrm{~mm}$. Resulting frequency responses and external acoustic noise infiltration curves are presented in Fig. 7.

Figure 7a shows that the external duct diameters influence the frequency response of the cell only in the range of low frequencies (below $200 \mathrm{~Hz}$ ), which is negligible taking into consideration that in the discussed cell the light beam would be modulated with a frequency of at least a few hundreds of $\mathrm{Hz}$ (preferably at $f_{0}$, as already mentioned). 

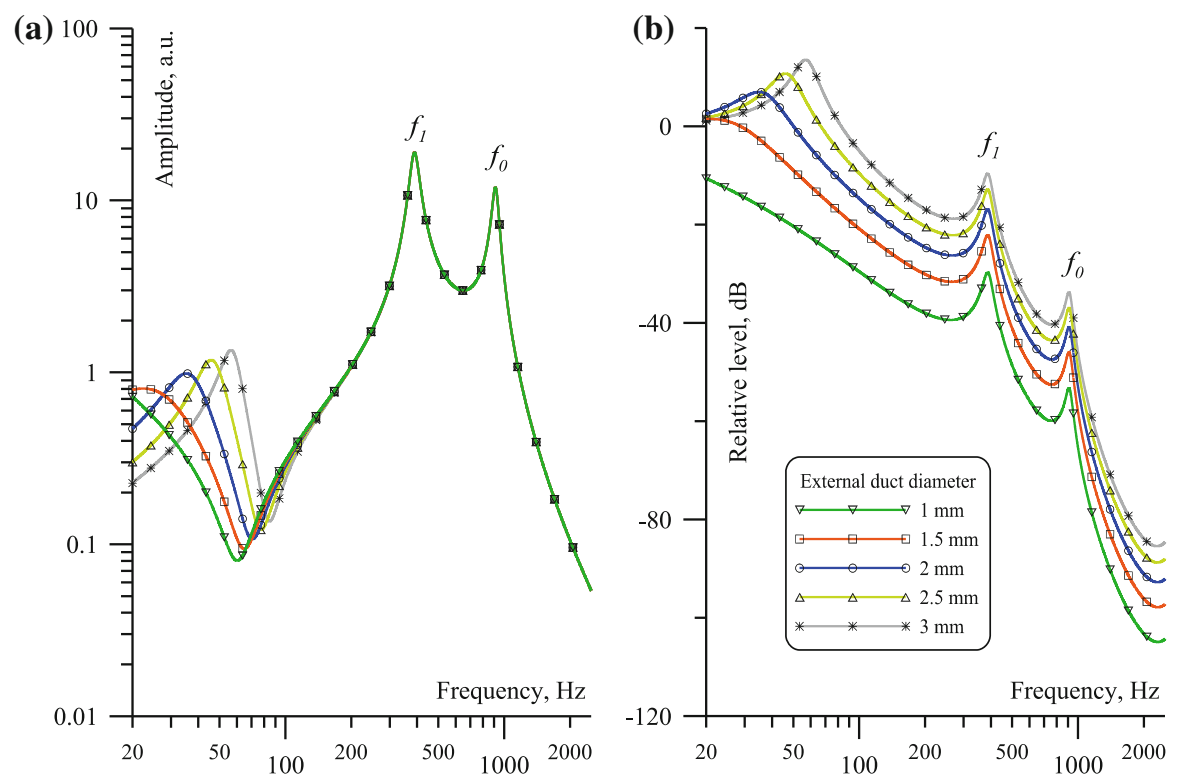

Fig. 7 Influence of the external duct diameter on (a) the frequency response and (b) external acoustic noise infiltration of the cell

As predicted, a reduction of the diameter of the external ducts increases attenuation of the external acoustic noise (Fig. 7b).

Similarly, as in the case of the diameter, a change of the length of the external ducts does not affect the frequency response above $200 \mathrm{~Hz}$, in particular, at the resonances $f_{0}$ and $f_{1}$ (Fig. 9a), but it has a strong influence on the external acoustic noise penetration (Fig. 8b). In the case of frequencies below $f_{0}$, longer ducts result in greater noise attenuation. For the frequencies above the main resonance and when the length is greater than some optimal value, which in this case is a few tens of millimeters, the attenuation of the noise is worse due to additional resonances approaching $f_{0}$.

As was predicted, buffer volumes help to minimize the noise penetration. The greater is the volume, the higher is the attenuation (Fig. 9b). However, there is a value above which further enlargement of the buffers does not result in substantial improvement of the external acoustic noise attenuation. Even at relatively low values (10 $\mathrm{cm}^{3}$ compared to $2 \mathrm{~cm}^{3}$ of the sample cavity), buffer volumes do not affect the frequency response in the proximity of $f_{0}$ (Fig. 9a).

\section{Conclusions}

The presented parametric analysis showed that many of the mechanical dimensions of the improved open Helmholtz cell can be adjusted in a relatively wide range virtually without affecting the frequency response around the resonance $f_{0}$ created by the main Helmholtz structure (interconnected sample and microphone cavities). A reduction of the diameter of the external and internal ducts results in a decrease of the noise 

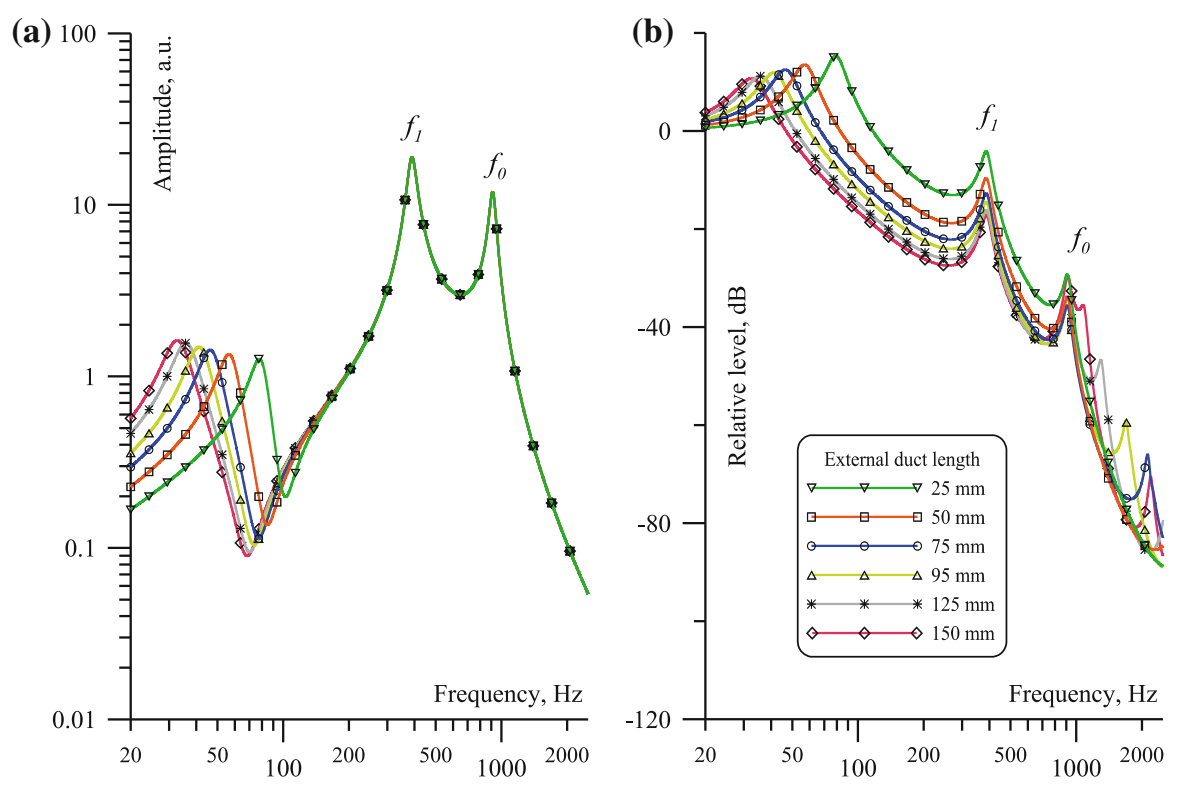

Fig. 8 Influence of the external duct length on (a) the frequency response and (b) external acoustic noise infiltration of the cell
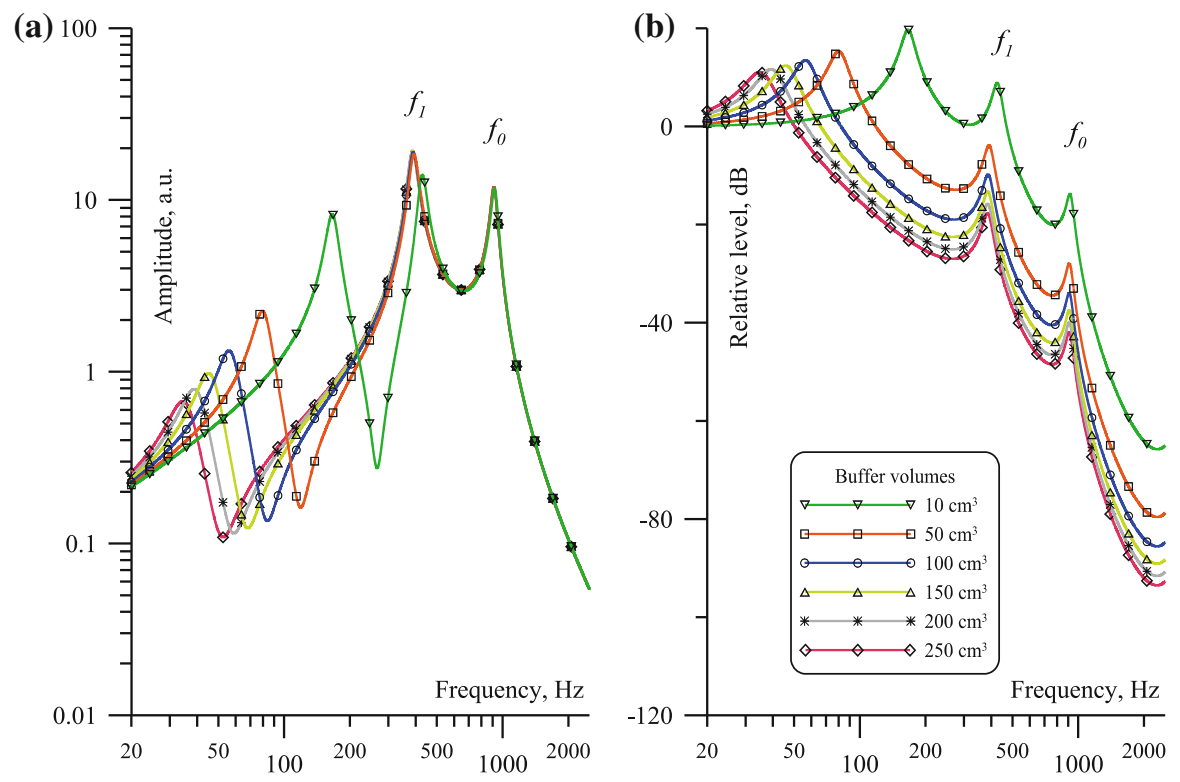

Fig. 9 Influence of the buffer volumes on (a) the frequency response and (b) external acoustic noise infiltration of the cell

infiltration. The length of the internal ducts should be appropriately selected in order to maximize the $Q$-factor of the cell and minimize external acoustic noise infiltration. Shorter external ducts may have better noise attenuation above the mentioned above 
resonance frequency $f_{0}$, but have worse properties in the range of frequencies below the resonance. The optimal length of the ducts is equal to a quarter-wave length of the modulation light. Use of buffer volumes of at least $100 \mathrm{~cm}^{3}$ and careful selection of the duct dimensions should attenuate the external acoustic noise by at least $50 \mathrm{~dB}$.

Open Access This article is distributed under the terms of the Creative Commons Attribution License which permits any use, distribution, and reproduction in any medium, provided the original author(s) and the source are credited.

\section{References}

1. D.H. McQueen, J. Phys. E Sci. Instrum. 16, 738 (1983)

2. M.D. da Silva, I.N. Bandeira, L.C.M. Miranda, J. Phys. E Sci. Instrum. 20, 1476 (1987)

3. J.A. Balderas-López, A. Mandelis, J. Appl. Phys. 90, 2273 (2001)

4. A. Keller, M. Rüegg, M. Forster, M. Loepfe, R. Pleisch, P. Nebiker, H. Burtscher, Sens. Actuators B 104, 1 (2005)

5. Z. Bozóki, A. Szabó, Á. Mohácsi, G. Szabó, Sens. Actuators B 147, 206 (2010)

6. T. Starecki, Acta Phys. Pol. A 114, A199 (2008)

7. T. Diószeghy, A. Miklós, A. Kelemen, A. Lörincz, J. Appl. Phys. 58, 2105 (1985)

8. T. Starecki, Acta Phys. Pol. A 114, A211 (2008)

9. T. Starecki, A. Geras, Int. J. Thermophys. (2013). doi:10.1007/s10765-013-1479-y

10. T. Starecki, Electron. Telecommun. Q. 39, 307 (1993)

11. M. Suchenek, Proc. SPIE 6937, 693711 (2007)

12. T. Starecki, J. Acoust. Soc. Am. 122, 2118 (2007)

13. T. Starecki, Proc. SPIE 6159, 61592M-1 (2006) 